\title{
Detection of Suspicious of Diabetic Feet using Thermal Image
}

\author{
Brian Meneses-Claudio ${ }^{1}$, Witman Alvarado-Díaz ${ }^{2}$, Fiorella Flores-Medina ${ }^{3}$ \\ Natalia I. Vargas-Cuentas ${ }^{4}$, Avid Roman-Gonzalez ${ }^{5}$ \\ Senior Member, IEEE ${ }^{5}$ \\ Image Processing Research Laboratory (INTI-Lab) ${ }^{1,2,3,4,5}$ \\ Universidad de Ciencias y Humanidades, Lima, Perú
}

\begin{abstract}
Diabetic foot is a chronic disease that occurs due to increased glucose levels, in addition to being the result of poorly controlled diabetes. In this case, the affected foot increases in temperature, because it contains accumulated blood. According to the Alianza para el Slvataje del Pie diabético en el Perú reported that currently has been increasing the cases of diabetic foot being $8 \%$ of the Peruvian population suffering from diabetic foot. Many research papers mention that the temperature difference of both feet has to be minimal due to the homogeneous distribution of the body, but when the temperature of the feet is higher than $2.2^{\circ} \mathrm{C}$ degrees with respect to the other foot, it is an indicative of diabetic foot. That is why the thermal evaluation of feet with suspected diabetic foot was raised in this research to prevent future damage or even amputation of the foot; first a thermal image of both feet is captured using the FLIR ONE Pro thermal camera following a temperature range protocol, then the images are processed in the MATLAB software in order to obtain the zones where the variations are greater or equal 2.2 degrees of temperature and finally superimpose it on the foot with higher temperature to determine the area where the highest temperature was detected. It was obtained as results, that patients with diabetic foot do not have sensitivity in both feet, which will indicate us as a result and in addition to the difference in temperature between both feet which is a possible diabetic foot.
\end{abstract}

Keywords-Diabetic foot; thermal images; image processing; Roberts method; heat map

\section{INTRODUCTION}

Diabetic foot is considered a chronic disease, this is caused by the increase of glucose (sugar) levels in the blood [1]. This increase is due to the absence of insulin secretion, insulin is secreted by the pancreas that helps control glucose, meaning that if there is no insulin, and glucose stays in the blood for a long time causing serious problems in the blood health.

According to the National Institute of Diabetes and Digestive and Kidney Diseases (NIH) and the Alianza para el Slvataje del Pie diabético en el Perú indicate that around the world every 20 seconds a limb is amputated and most of these cases are due to the diabetes, because it is a silent disease and currently in Peru affects $8 \%$ of the Peruvian population. In $25 \%$ of patients with diabetic foot, it can start as a callus or a small wound that does not heal because of diabetes, therefore, it causes an infection that is happening inside [2].
The diabetic foot is an alteration to the nerves and indicated by hyperglycemia (high sugar) [1], in which with or without coexistence of ischemia, produces injury and / or ulceration of the foot called diabetic foot. In addition, it is also considered hereditary because in case the patient has relatives with diabetics and diabetic foot means that he or she is prone to suffer from the same evils; that is why doctors always identify these risks, recommend care and prevention of sugars.

The causes of diabetic foot injuries are peripheral neuropathy which is the loss of sensation in the foot, means that you do not feel any pain, in order to not perceive the injuries that can be caused internally and the vasculopathy that is known as the lack of blood flow [3], this is due to the constant increase in blood glucose causes some small blood vessels that irrigate the foot to narrow causing the oxygen and essential nutrients not reach complete to the foot, by conclusion, due to the decrease in blood supply, makes the wounds take longer to heal and be of high risk when infected [4].

In [5], a comparative map was proposed between patients with and without diabetic foot using infrared thermography, 479 subjects were taken of whom 277 people had diabetic foot with a mean age of 63.41 years, having as a result the difference of different areas of the soles of the feet bilaterally, also reached the conclusion that the use of infrared thermography is important as an evaluation method for the risk of diabetic foot, thus giving diagnosis and prevention in areas where Higher temperature index is identified.

In [6], they indicate that used the FLIR ONE to capture images of the soles of the feet to identify diabetic foot on the basis of a temperature difference of 0.987 and 0.0981 , and after they processed the images, they clinically proved that the results obtained were from patients with diabetic foot. In this case, in this research work they want to have thermal cameras in the health centers for the verification and validation of diabetic foot in an instantaneous way and thus diagnoses about the care that these patients should have.

The main objective of the research work is the detection of suspicions of diabetic foot using thermal images to prevent future amputations because it is a disease that has as a consequence harsh repercussion. This detection is due to the processing of images where we will show if the patient has a 
temperature difference greater than $2.2{ }^{\circ} \mathrm{C}$ [7], which is considered as an abnormal temperature variation between both feet.

Thermography is the study of the variation of temperature, that is, it identifies exact temperatures of a body and objective without the need of physical contact, just by pointing with the thermography analyzer equipment, it has as purpose the study of said signals for the detection of evils [8] where temperature is an important and / or decisive factor. The equipment makes use of thermography can be modified based on the temperature range to be measured, one of the best known are the FLUKE or FLIR ONE Pro [9], equipment, due to its compatibility with the Android and IOS systems. Currently, there are thermal cameras that connect to Smartphone and are used in various areas of research.

The thermal images show the thermal composition of a body or objective, in addition to identifying in which areas there is a higher temperature, also, the thermal cameras have software in which the images can be processed to provide accurate data of the pixels of the image [10]. These thermal images have many applications being one of the most necessary in the field of medicine because the temperature variation of the human body is a key to the development of the same and the detection of diseases or internal sutures.

The following research work is structured as follows: In section II, the development of the processing of thermal images of the possible diabetic foot will be presented. In Section III, the results will show the thermal image of the feet and the image of the warmer foot with the superimposed heat map indicating the temperature zones greater than $2.2^{\circ} \mathrm{C}$. In Section IV, we present the discussions of the research work and finally in Section $\mathrm{V}$, the conclusions, as well as the future work that is to be achieved with the research work.

\section{Methodology}

In this part, each part of the segmentation of the thermal image is developed for the detection of suspicions of diabetic foot, which consist of the acquisition of the image, processing of the image and finally the results that correspond to the segmented image superimposed on the real image. The stages of the system are shown in Fig. 1 where the processes of the thermal image will be subjected are indicated.

\section{A. Image Acquisition}

For our research, it requires a camera capable of capturing thermal images, for this reason, it uses the FLIR ONE Pro thermal camera, which is compatible with Android and IOS mobile devices. In addition, it has its own application for mobile devices and software to process the thermal images stored in the device that is connected. This device has two lenses as shown in Fig. 2, the upper lens captures digital images and the lower lens captures the thermal images that will be stored in the mobile device that is connected. In this case, an Android mobile device was used then a $\mathrm{C}$ to Micro USB connector was required because the thermal camera has a $\mathrm{C}$ connector from factory.

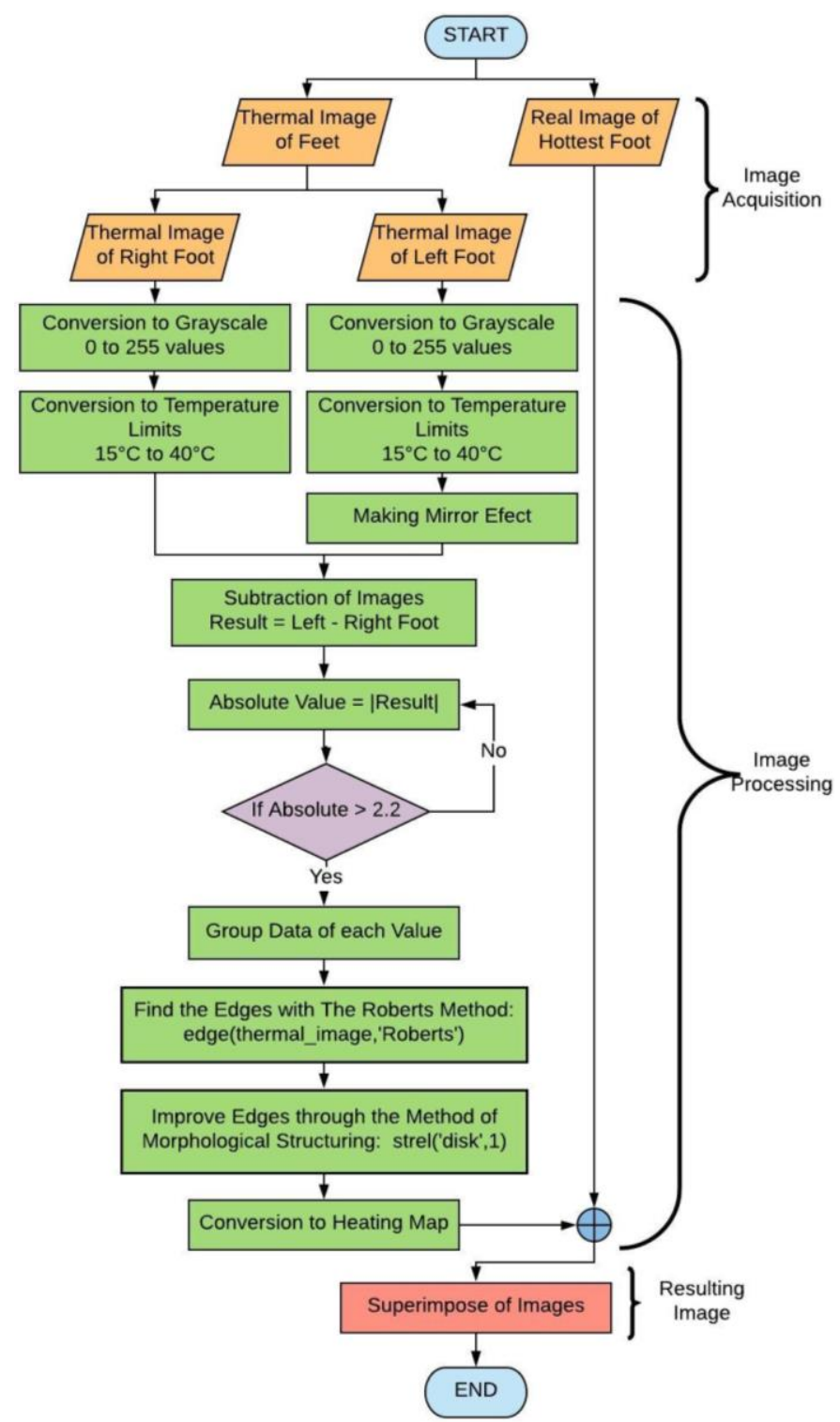

Fig. 1. Diagram of Flow for the Detection of Suspected Diabetic Foot.

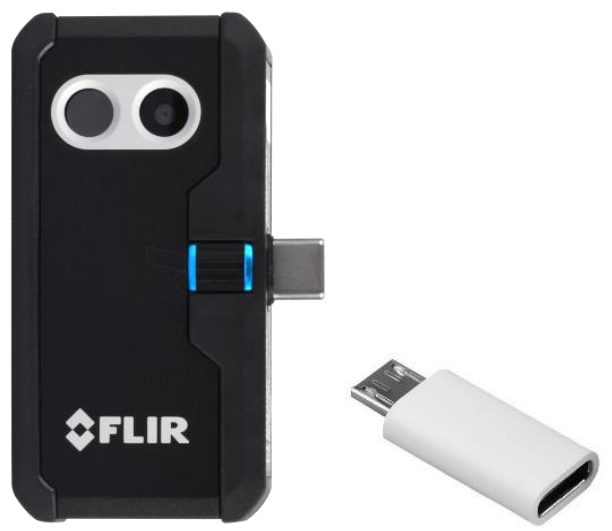

Fig. 2. FLIR ONE Pro Thermal Camera and Type $\mathrm{C}$ to Micro USB Connector Adapter. 
TABLE I. CHARACTERISTICS OF THE FLIR ONE PRO

\begin{tabular}{|c|c|}
\hline \multicolumn{2}{|l|}{ FLIR ONE Pro } \\
\hline Temperature Range & $-20^{\circ} \mathrm{C}-400^{\circ} \mathrm{C}$ \\
\hline Compatibility & IOS y Android devices \\
\hline Distance & 1.8 meters \\
\hline Weight & $36.5 \mathrm{~g}$. \\
\hline Dimensions & $68 \times 34 \times 14$ millimeters \\
\hline Thermal Resolution & $160 \times 120$ \\
\hline Operating Time & 1 hour \\
\hline
\end{tabular}

The thermal camera has characteristics in which its images and operation are governed; these characteristics are shown in Table I.

The FLIR ONE Pro thermal camera is an electronic device whose main function is to capture images in temperature ranges, in its principles, it was used to identify faults in industrial equipment and improve repair time, at present, they are used in several fields in which one of the requested is to medicine due to the variation of temperature as detection of internal and / or pathological evils; as in this case, detection of suspicion of diabetic foot.

\section{B. Image Processing}

The images obtained are processed to identify the points where the highest temperature variation indexes are observed as shown in Fig. 3, where we observe the real image, then the thermal image, the subtraction of both images identifying the heat zones, and finally superimposed on the foot with higher temperature.

The MATLAB Software reads the images using FLIR ONE Pro, each thermal image is composed of pixels in 3 dimensions because it is in RGB form, so, it will convert this image to gray scale. It has to take into account, that they are 2 foot and it must process both for this, the software makes a weighted sum taking the values of each pixel of the image of each dimension and multiplying with a normed value as shown below:

$0.2989 * R+0.5870 * G+0.114 * B$

After converting both feet to gray scale that is within the range of $0-255$ in two dimensions, we have to convert it to a temperature scale.

To convert gray scale images to a temperature scale, we have to map the image and therefore using the following formula:

Map $=(($ Pixel_Image - FromLow $) *($ ToHigh - ToLow $) /$

(FromHigh - FromLow) $)+$ ToLow

Where:

- FromLow y FromHigh = 0 y 255 (Gray Scale)

- ToLow y ToHigh $=$ Temperature Range to which we will convert the image.

Replacing formula 2, we will put the values to which we want the image, as shown in the following formula:

output1 $=(($ Pixel_Image -0$) *(40-15) /(255-0))+15$
We do the following process for both images to obtain them in temperature range, then it applies Mirror method to the coldest foot so it is in the same position as the warmer foot, we do this process because the MATLAB software will proceed with the subtraction of each pixel, thus identifying the hottest areas. For the subtraction of the images we use a simple subtraction.

Subtraction $=$ Thermal_After-Thermal_Before

According to [4], they explain that the temperatures of both feet can have a minimum temperature range of up to $1.4^{\circ} \mathrm{C}$ but when the temperature difference is greater than $2.2^{\circ} \mathrm{C}$, also when is resting for more than 20 minutes, it is an indicative of diabetic feet [7]. That is why after obtaining the subtraction of both images, it sectorizes the pixels where there is a difference greater than $2.2^{\circ} \mathrm{C}$, as shown in Fig. 1, in the flow diagram.

It is indicated that when the pixel meets the aforementioned value, the data will be grouped and will show only the points that exceed the value of $2.2^{\circ} \mathrm{C}$.

After obtaining the zones with the highest temperature, it will identify the edges, for this case, the Roberts Method was used because it is the only method of edge detection of morphologically modified images, meaning that they do not have a specific shape. Roberts' method uses a filter that focuses on each pixel through the following formula:

$\frac{d f}{d x}=f(x+1, y)-f(x, y)$

$\frac{d f}{d y}=f(x, y+1)-f(x, y)$

Where to locate the pixel $(\mathrm{x}, \mathrm{y})$ that is in gray scale within the range of 0 to 255, if the areas have a constant intensity, will turn them into 0 .

After obtaining the edges, as it knows that the image does not have a specific shape, in the same way the edges will be distorted, therefore, a filter was used to improve the segmentation called Morphological Structure Method as shown below:

$\delta_{B}(X)=X \oplus B=\left\{x \mid X \cap B_{x} \neq \emptyset\right\}$

Where it is indicated that $\mathrm{X}$ will travel through the whole image, when it passes through $\mathrm{B}$, it will give the information of the data of the neighbors of that pixel, converting it to the maximum value of the environment of that neighborhood defined by the element of the structure. The values that are around each pixel are called neighborhood. Then when the pixel has a neighborhood of values different from it, it will take the maximum value of that neighborhood.

Finally, all the images obtained as the edges, the image of the subtraction of the thermal images and the real image will be superimposed, to obtain the image with the zones where there is a temperature difference of $2.2^{\circ} \mathrm{C}$ being an indicative of suspicions of diabetic foot, as shown in Fig. 3(d). 


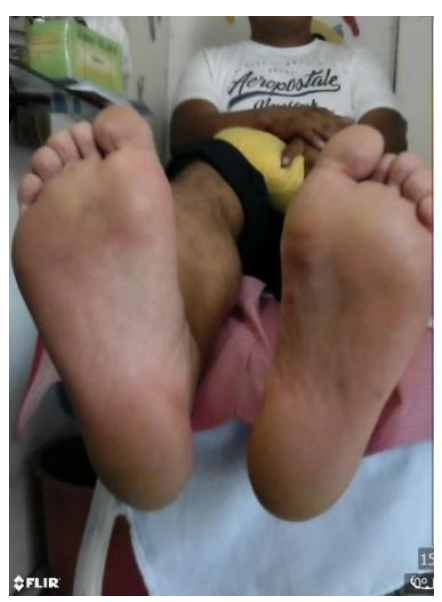

(a)

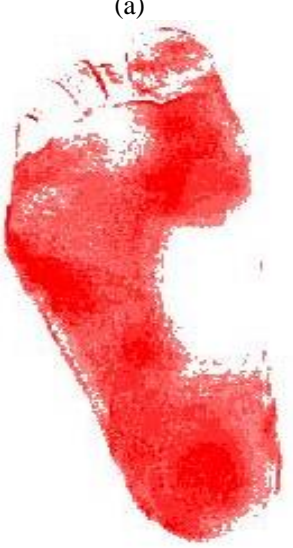

(c)

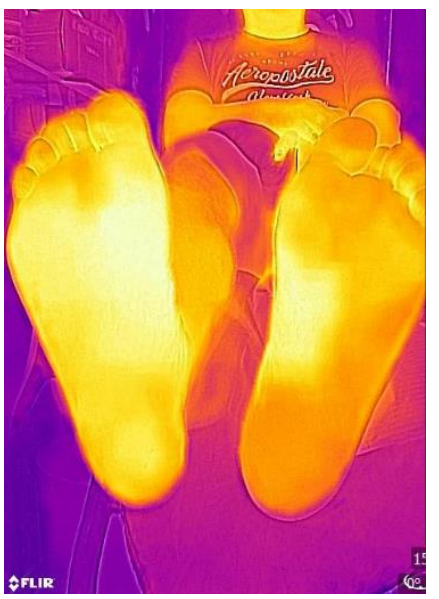

(b)

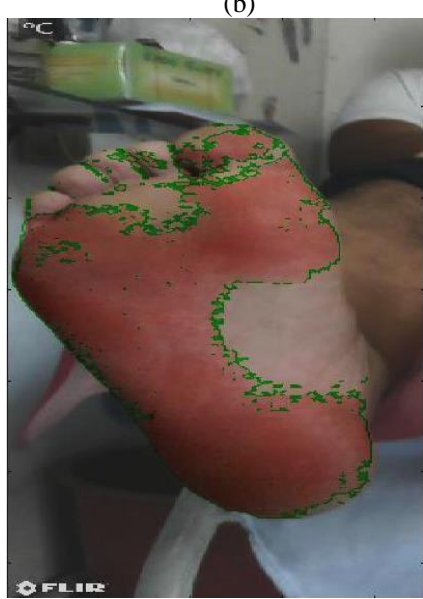

(d)

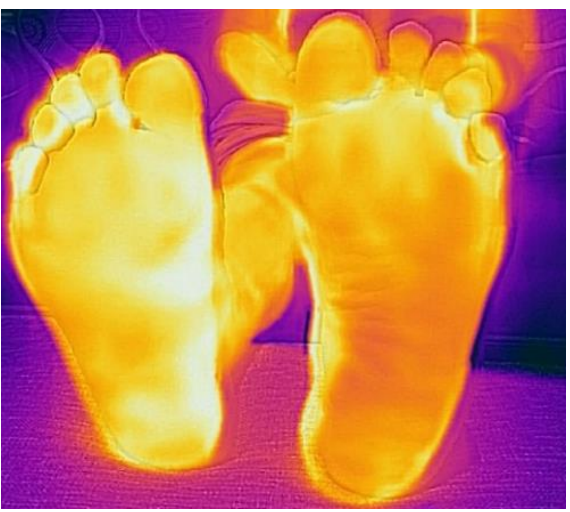

(a)

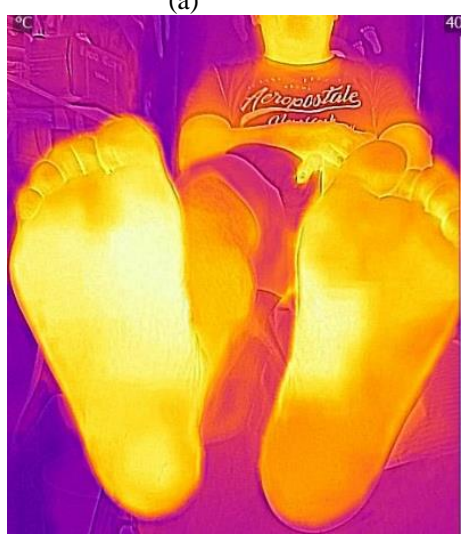

(a)

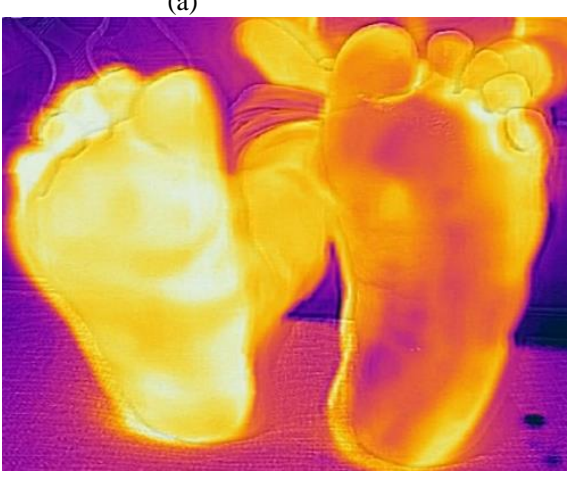

(a)

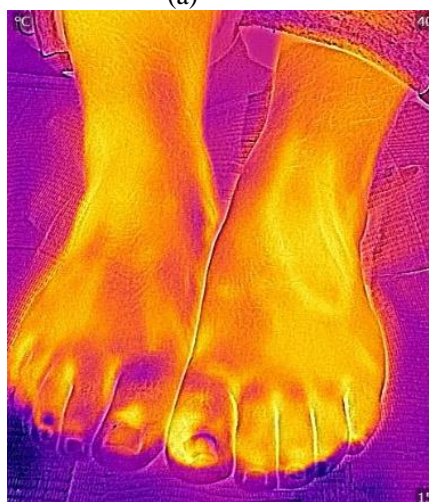

(a)

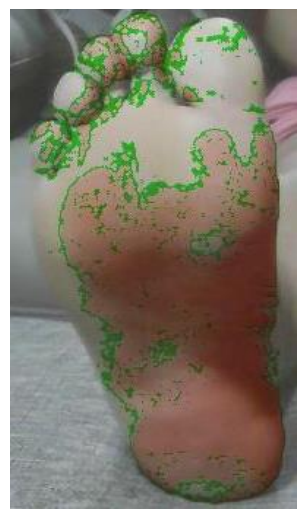

(b)

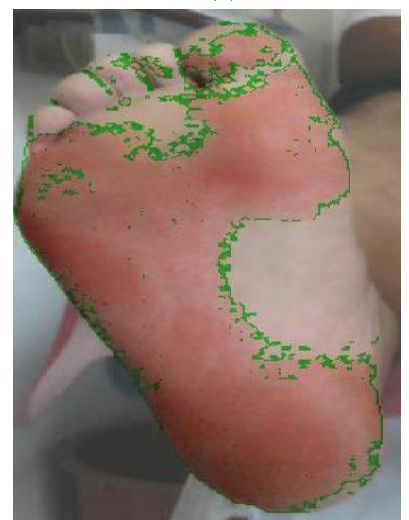

(b)

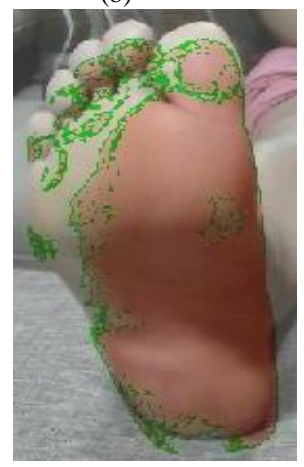

(b)

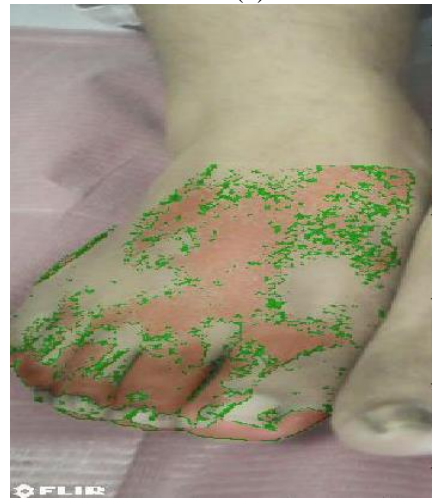

(b)

Fig. 4. (a) Thermal Images of the Feet. (b) The Superposition of the Zone with a Temperature Greater than $2.2{ }^{\circ} \mathrm{C}$ on the Real Image. 


\section{DISCUSSION}

The research work confirms the use of thermal images for the detection of suspicion of diabetic foot due to the variation of temperature in the feet.

For the image processing, the real image and the thermal image were required because at the end of the process it was necessary to superimpose the segmented area of the suspicion of diabetic foot to the real image.

Many patients are alarmed when identifying that they have diabetic foot; that is why we indicate that this research work is a preliminary work to detect suspicions of diabetic foot, because there are many factors being food one of the most relevant.

This study is for the prevention of the diabetic feet, we are not considering the clinic historical so this is study has more variable to take into consideration.

\section{CONCLUSIONS}

It is concluded that a possible diabetic foot can be detected through thermal images in an efficient and fast way because it only requires capturing the images and then the software will segment it automatically obtaining the area where is the possibility of having a case of diabetic foot.

It is concluded that the podiatry center provides relevant information for the thermal image processing and that it complements the protocol with the rest period to regularize the internal temperature of the patients' feet.

As work in the future, it is necessary to detect diabetic foot in short time so that patients follow a healing process, besides that externally it is not possible to identify if a foot is diabetic that is why through thermal imaging you can know if you have signs or suspicious of diabetic foot.

\section{REFERENCES}

[1] Uçkay, J. Aragón-Sánchez, D. Lew, and B. A. Lipsky, "Diabetic foot infections: what have we learned in the last 30 years?,' Int. J. Infect. Dis., vol. 40, pp. 81-91, Nov. 2015.

[2] S. Seclén, "Diabetes Mellitus en el Perú: hacia dónde vamos," Rev. Medica Hered., vol. 26, no. 1, pp. 3-4, 2015.

[3] Y. García García, E. Hernández Lao, A. Hernández Soublet, J. A. Barnés Domínguez, and Z. Durán Balmaseda, "Therapeutic education on diabetes for patients with first amputation caused by diabetic foot," Rev. Cuba. Angiol. y Cirugía Vasc., vol. 17, no. 1, pp. 0-0, 2000.

[4] J. Federico, R. Cruz, E. Bonilla Huerta, R. Cocoletzi, and J. Crispín Hernández Hernández, "Advances in the Development of a Thermographic Image Classifier of Diabetic Foot Plant Based on Backpropagation Neural Network," 2016.

[5] Á. Astasio-Picado, E. Escamilla Martínez, and B. Gómez-Martín, "Mapa térmico comparativo del pie entre pacientes con y sin diabetes mediante el uso de termografía infrarroja," Enfermería Clínica, Jan. 2019.

[6] R. F. M. van Doremalen, J. J. van Netten, J. G. van Baal, M. M. R. Vollenbroek-Hutten, and F. van der Heijden, "Validation of low-cost smartphone-based thermal camera for diabetic foot assessment," Diabetes Res. Clin. Pract., vol. 149, pp. 132-139, Mar. 2019.

[7] C. Liu, J. J. van Netten, J. G. van Baal, S. A. Bus, and F. van der Heijden, "Automatic detection of diabetic foot complications with infrared thermography by asymmetric analysis," J. Biomed. Opt., vol. 20, no. 2, p. 026003 , Feb. 2015.

[8] R. Vardasca, L. Vaz, C. Magalhães, A. Seixas, and J. Mendes, "Towards the Diabetic Foot Ulcers Classification with Infrared Thermal Images."

[9] V. Sagan et al., "UAV-Based High Resolution Thermal Imaging for Vegetation Monitoring, and Plant Phenotyping Using ICI 8640 P, FLIR Vue Pro R 640, and thermoMap Cameras," Remote Sens., vol. 11, no. 3, p. 330 , Feb. 2019.

[10] A. J. Singer, P. Relan, L. Beto, L. Jones-Koliski, S. Sandoval, and R. A. F. Clark, "Infrared Thermal Imaging Has the Potential to Reduce Unnecessary Surgery and Delays to Necessary Surgery in Burn Patients," J. Burn Care Res., vol. 37, no. 6, pp. 350-355, Nov. 2016.

[11] B. Meneses-Claudio, W. Alvarado-Diaz, and A. Roman-Gonzalez, "Detection of Suspicions of Varicose Veins in the Legs using Thermal Imaging," 2019. 